\title{
Cyclophilin A/CD147 Induces the Epithelial-to- mesenchymal Transition and Renal Fibrosis in Chronic Allograft Dysfunction by Regulating MAPK Signaling
}

\section{Xuzhong Liu}

Huai'an First People's Hospital, Nanjing Medical University

\section{Zhiwang Tang}

Huai'an First People's Hospital, Nanjing Medical University

\section{Xi Jiang}

Huai'an First People's Hospital, Nanjing Medical University

\section{Tianwei Wang}

Huai'an First People's Hospital, Nanjing Medical University

\section{Lun Zhao}

Huai'an First People's Hospital, Nanjing Medical University

\section{Zongyuan $\mathrm{Xu}$}

Huai'an First People's Hospital, Nanjing Medical University

Kun Liu ( $\nabla$ chenhao_nanjing@126.com )

Huai'an First People's Hospital, Nanjing Medical University

\section{Research Article}

Keywords: Cyclophilin A, CD147, epithelial-to-mesenchymal transition, kidney transplantation, chronic allograft dysfunction

Posted Date: March 18th, 2021

DOl: https://doi.org/10.21203/rs.3.rs-310337/v1

License: (c) (i) This work is licensed under a Creative Commons Attribution 4.0 International License. Read Full License 


\section{Abstract}

Objectives: Our study was designed to explore the role of Cyclophilin A (CyPA)/CD147 interactions in renal allograft fibrosis and chronic allograft dysfunction (CAD).

Methods and materials: A rat renal transplant model with significant CAD was successfully identified. Renal allograft tissues and blood samples were collected. HE, Masson and immunohistochemistry staining were performed. Then human HK-2 cells were intervened by certain concentrations of CyPA, and total protein and mRNA were extracted. Western blot assay and PCR were performed to explore the protein and mRNA expression of CyPA, CD147 and epithelial-to-mesenchymal transition (EMT)-related biomarkers. CD147 siRNA and specific inhibitor of MAPK were used to explore the involved cellular mechanism.

Results: We have successfully established and identified a 20-weeks renal transplant CAD model. We observed significant distributed and expressed CyPA and CD147 in the renal allograft fibrosis tissues. We also found the significant expression of CD147 and EMT-related markers in the HK-2 cells stimulated by CyPA. The CD147 siRNA confirmed the previous results in vitro. The selective inhibition of MAPK suggested the notable role of MAPK signaling pathway in the CyP/CD147 interactions involved in renal allograft fibrosis.

Conclusions: Our study reported the positive relationship of CyPA/CD147 interactions with the renal allograft dysfunction. In vitro study suggested that CyPA could bind to CD147 and then induce the development of EMT process by MAPK signaling, thus contributing to the renal allograft fibrosis and CAD.

\section{Introduction}

Chronic allograft dysfunction (CAD) remains to be the main factors influencing the long-term allograft survival of renal transplant recipients, which was characterized by interstitial fibrosis, tubular atrophy and glomerulitis $(1,2)$. While substantial studies have reported the mechanism of renal allograft fibrosis, there is still lack of detailed and comprehensive knowledge for the CAD. It is known that various factors, such as transforming growth factor-beta 1 (TGF- $\beta 1$ ), tumor necrosis factor- $a$ (TNF- $\alpha$ ), have been verified to play a crucial role in the formation of allograft fibrosis $(1,3,4)$. Recent years, Cyclophilin A (CyPA), an ubiquitous intracellular protein, has been recognized to be secreted into extracellular space by various cell types, such as macrophages, activated platelets (5). Moreover, extracellular CyPA exerts its inflammatory effects through binding to the Extracellular Matrix Metalloproteinase Inducer (EMMPRIN, CD147) in a celltype specific manner (6). It is well-documented that extracellular CyPA is currently involved in the expression of adhesion molecules and induction of the inflammatory responses, including the chemotaxis of monocyte, neutrophile granulocytes, T lymphocytes, and eosinophile granulocytes $(7,8)$.

Importantly, CyPA/CD147 is believed to be involved in the pathogenesis of fibrosis in several organs. The $B s g$, the name of mouse CD147 gene, promotes the transition of fibroblasts to myofibroblasts by 
increasing the expression of a-smooth muscle actin (a-SMA) in corneal fibroblasts (9). In cardiac model in mice, knockout of CyPA leads to the attenuation of macrophages and T lymphocytes infiltration, and also retards the progression of myocardiac fibrosis, indicating that CyPA may represent a pharmacological target to modulate the myocardial remodeling in myocarditis (10). Furthermore, knockout of Bsg is significantly related with the reduced production of matrix metalloproteinase (MMP) and induction of TGF- $\beta 1$, thus decreasing the formation of renal fibrosis (11). Therefore, interactions between CyPA and CD147 could be strongly suggested to be an important regulator of organ fibrosis, including renal fibrosis.

In this study, we hypothesized that CyPA/CD147 could protmote the progression of renal allograft fibrosis by targeting the epithelial-to-mesenchymal transition (EMT), which has been identified as a crucial origin of the renal fibrosis, consequently contribute to the CAD pathogenesis.

\section{Methods And Materials}

\section{Ethic statement}

The study protocol was in compliance with the principle of the Declaration of Helsinki and Istanbul. Whole study design, including animal and cell experiments, was approved by the ethics committee of Huai'an First People's Hospital.

\section{Animals and experimental design}

Adult male Lewis and F344 rats weighted ranging from 200 to $250 \mathrm{~g}$ were purchased from Charles River Laboratories (Beijing, China) and raised in the Animal Feeding Center of Nanjing Medical University with clean tap water and standard rat chow.

Orthotopic renal transplant surgery was conducted between the donor of Lewis rats and the recipient of F344 rats in CAD group. The mean warm ischemia time and cold ischemia time for the corresponding surgeries were 34.1 minutes (mins) and 15.9 mins. Nephrectomy surgery of right kidney in recipient rats was performed after the 3 days post-transplant. Cyclosporine A with a concentration of $5 \mathrm{mg} / \mathrm{kg}$ bodyweight (Novartis, Switzerland) was administrated once a day intraperitoneally, which lasted for 14 days post-transplant. The sham surgery was performed on the F344 rats from the same batch, which was considered as sham group. The recipients were executed by head-breaking after 20 post-transplant weeks, and renal allograft tissues were harvested. Then, samples were divided into two parts, which were conserved in $-80^{\circ} \mathrm{C}$ liquid nitrogen and fixed in the paraffin, respectively. In addition, the blood samples of recipient rats in both groups were collected in the 4,12 and 20 post-transplant weeks, and stored in $-80^{\circ} \mathrm{C}$ liquid nitrogen. Concentrations of serum creatinine and blood usea nitrogen (BUN) were assessed according to the manufacturer's instructions (Jiancheng, Beijing, China). 


\section{Histology}

The collected rat renal allograft tissues were used for the HE, Masson trichome and immunohistochemistry (IHC) staining as described previously (12). Briefly, 2- $\mu \mathrm{m}$ sections were stained with Haematoxylin and Eosin during the HE staining. The morphological changes were observed and evaluated by interstitial fibrosis and tubular atrophy, and fibrosis area was calculated from five fields of view in each section. Furthermore, sections were de-paraffinized and rehydrated in a series of alcohol with graded concentrations. The primary antibodies (rabbit polyclonal anti-E-Cadherin, 1: 100; rabbit polyclonal anti-a-SMA, 1: 200; rabbit polyclonal anti-CD147, 1: 200; rabbit polyclonal anti-CyPA, 1: 200; Abcam, USA) were used to incubate with sections overnight at $4{ }^{\circ} \mathrm{C}$. Analysis of positive staining was performed under a light microscope equipped with digital cameras (Nikon, ECLIPSE 80i, Japan) by two independent authors (K Liu and XZ Liu).

\section{Cell culture, treatment and transfection}

HK-2 cell line was purchased from the KeyGen Biotech Company (Nanjing, China), and cultured in the Dulbecco's modified Eagle's medium (DMEM)/F12 medium containing 1\% penicillin-streptomycin and $10 \%$ Fetal Bovine Serum (FBS) in certain atmosphere with $5 \% \mathrm{CO}_{2}$ at $37^{\circ} \mathrm{C}$. For the CyPA intervention, starvation of HK-2 cells was performed without any serum overnight and stimulated with various concentrations of CyPA $(100 \mu \mathrm{g} / \mathrm{ml}, 200 \mu \mathrm{g} / \mathrm{ml})$ for 5 days, whereas the control group was treated with the solution of CyPA. At the intensity of approximately $80 \%$, cells were transfected with siRNA-CD147 plasmid (50nM; KeyGene BioTECH, Nanjing, China) by using Lipofectamine 2000 (Invitrogen, USA) according to the manufacture's procedures. Cells were treated as described previously after the transfection for $6 \mathrm{~h}$. For the MAPK-specific inhibitor, SB203580 $(10 \mu \mathrm{M})$, was applied ahead to HK-2 cells for 30 mins before the intervention of CyPA and/or siRNA.

\section{Real-time PCR}

Rat kidney tissues were snap-frozen in liquid nitrogen for total mRNA isolation, whereas total mRNA of HK-2 cells from each group was also extracted by the RNA extraction kits (TIANGEN, Beijing, China). Briefly, a PrimeScriptTMRT reagent kit (TaKaRa Biotechnology, Japan) was used to synthesize the cDNA. The quantitative real-time PCR (qRT-PCR) was conducted by a SYBR Green PCR kit (TaKaRa Biotechnology) under a DNA Engine Opticon 2 System (BioRad laboratories, Hercules, CA). The primers used for the mRNA evaluation were shown as follows: CD147, 5'GGAATTCGCCACCATGGCGGCTGCGCTGTTCG-3 ' (F) and 5'-CGGGATCCTCAGGAAGAGTTCCTCTGG-3' (R) ; CyPA, 5'-CAAGGTCCCAAAGACAGCAGA-3' (F), 5'-AAG ATG CCA GGA CCC GTA TGC-3' (R) ; E-Cadherin, 5'-CGAGAGCTACACGTTCACGG-3' (F), 5'-GGGTGTCGAGGGAAAAATAGG3' (R); Fibronectin (FN), 5'CGGTGGCTGTCAGTCAAAG-3'(F), 5'-AAACCTCGGCTTCCTCCATAA-3'(R); Actin: 5'- 
TGACGTGGACATCCGCAAAG-3' (F), 5'-CTGGAAGGTGGACAGCGAGG-3' (R). All the experiments mentioned were carried out for at least three times.

\section{Western Blotting}

The detailed procedures of Western Blot assay were described as previously reported (12). Briefly, total proteins were extracted from the rat kidney tissues and HK-2 cells derived from each group. Primary antibodies used in this study were shown as below: anti-a-SMA antibody (1:1000; Abcam, USA); anti-ECadherin antibody (1:1000; Abcam, USA); anti-FN antibody (1:1000; Abcam, USA); anti-CD147 antibody (1:1000; Abcam, USA); anti-CyPA antibody (1:1000; Abcam, USA); anti-GAPDH antibody (1:1000; Abcam, USA); anti-phospho-p38 MAPK antibody (1:1000; Cell Signaling Technology, USA); anti-p38 MAPK antibody (1:1000; Cell Signaling Technology, USA). The secondary antibody in this study was goat antirabbit IgG (1:4000; Abcam, USA). The proteins were visualized by chemiluminescence reagents (Amersham Biosciences, USA). All the experiments above were carried out for at least three times.

\section{Enzyme-linked immunosorbent assay (ELISA)}

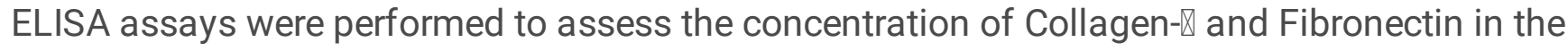
supernatant of cell culture treated with siRNA-CD147 and/or CyPA. The whole procedures were performed as described by the manufacturer's instructions. The assays were performed for at leat three times.

\section{Statistical analysis}

All data was shown as mean \pm standard deviations (SD). Statistical analysis was conducted with the unpaired, two-tailed Student's $t$-test for single comparisons. A P value less than 0.05 was considered as statistical significant. All statistical analysis was performed by SPSS v18.0 (SPSS Inc., Chicago, IL, USA).

\section{Results}

\section{CyPA and CD147 are highly expressed in chronic allograft dysfunction}

We have established a 20-weeks rat renal transplant model with CAD. As shown in Figure 1, remarkable interstitial fibrosis and tubular atrophy (IF/TA), which was considered as the crucial pathological characteristics for $C A D$, was observed in the HE and Masson staining from allograft in the CAD group (Figure 1A-B). Moreover, significant infiltration of mononuclear cells among the IF/TA was also found. The quantitative analysis of fibrosis area between two groups supported the identification of rat renal transplant CAD model (Figure 1C). Consequently, we analyzed the allograft function by serum creatinine 
and blood urea nitrogen (BUN), and results showed that renal allograft function was significantly reduced in a time-dependent manner in CAD group when compared with the sham subjects (Figure 1D-E).

We tested protein expression of E-Cadherin, as well as a-SMA, the biomarkers of epithelial-tomesenchymal transition (EMT), in the allograft tissues. The results showed that the expression of a-SMA was up-regulated and expression of E-Cadherin was decreased, suggesting the existing presence of EMT progression in CAD (Figure 2A-D). Then, we designed to explore the protein distribution and expression of CyPA and CD147 in the pathogenesis of CAD. Enhanced expression of CyPA and CD147 were identified in 20-weeks renal allograft tissues and mainly distributed in the allograft tubular epithelial regions (Figure $2 \mathrm{E}-\mathrm{H}$ ). Furthermore, we examined the total protein and mRNA expression of CyPA and CD147 in allograft tissue. The results were consistent with those in IHC staining (Figure 2I-K). Thus, total results suggested the potential association of increased CyPA and CD147 with the progression of EMT and renal allograft fibrosis.

\section{CyPA/CD147 interaction induces the progression of EMT}

To clarify the mechanism of CyPA/CD147 during the EMT progression and renal fibrosis, in vitro experiments were performed. Western blotting results showed the protein expression of CD147, as well as a-SMA and Fibronectin (FN), were remarkably higher after the stimulation of CyPA in a dose-dependent manner in HK-2 cells, whereas the expression of E-Cadherin was conversely reduced (Figure 3A). Similar results were indicated by the mRNA expression (Figure 3B-D). Next, we silenced the expression of CD147 in HK-2 cells to further confirm the effect of CyPA on the EMT progression. The siRNA-CD147 intervention significantly reduced the expression of CD147 in HK-2 cells, whereas the positive regulation of a-SMA and

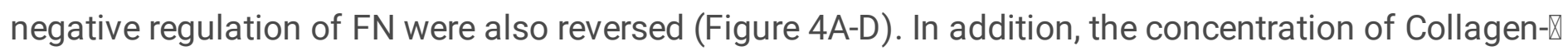
and FN in the supernatant were assessed by ELISA assay. The results showed that secretion of Collagen$\triangle$ and FN from the HK-2 cells induced by CyPA were significantly inhibited by siRNA-CD147. Therefore, the in vitro study indicated that the CyPA/CD147 interaction could significantly induce the progression of EMT in HK-2 cells.

\section{MAPK signaling pathway contributes to the progression of EMT and renal fibrosis induced by CyPA/CD147 interaction}

It's reported that MAPK signaling pathway participates in the regulation of CyPA and CD147 (13). Hence, we further explored the potential mechanism involved in the effect of CyPA/CD147 interaction on the progression of EMT and renal fibrosis. Firstly, we tested the activation of MAPK signaling in the CyPA/CD147 interactions. Figure 5A showed significant activation of phosphorylated p38 MAPK, the crucial mediator of MAPK signaling, of which could be significantly inhibited by the treatment of siRNACD147 (Figure 5B). Then, we used the specific inhibitor for MAPK signaling (SB203580), to confirm the activation of MAPK signaling. We have observed that upon the intervention of SB203580, the expression 
of CD147 showed no significance (Figure 5C-D), whereas the expression of a-SMA was remarkably inhibited (Figure $5 \mathrm{C}$ and $5 \mathrm{E}$ ), suggesting the downstream role of MAPK signaling among the interaction of CyPA/CD147. Importantly, the activation of phosphorylated p38 MAPK was also observed in the renal allograft tissue from rat renal transplant CAD model (Figure 5F-G).

\section{Discussion}

Our in vivo and vitro study showed that CyPA/CD147 interactions were significantly activated in the progression of renal allograft fibrosis and CAD. Moreover, CyPA/CD147 could remarkably induce the process of EMT by MAPK signaling and then contribute to the renal allograft fibrosis, indicating the CyPA/CD147 could serve to be an important target for the renal allograft fibrosis. To the best of our knowledge, this is the first study to investigate the role of CyPA/CD147 interactions in the renal allograft fibrosis.

Numerous reports were designed to investigate the cellular mechanism of CAD in renal transplant, factors of which could be divided into immunological and non-immunological factors (14). Among these, EMT was identified to play a crucial role in the pathogenesis of renal allograft fibrosis by inducing the

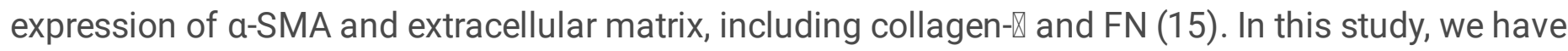
identified that EMT induced by CyPA/CD147 contributes to the renal allograft fibrosis, which was consistent with the specific role of EMT in renal fibrosis. Also, blockade of CD147 could also attenuate the progression of EMT and renal fibrosis, suggesting the downstream effect of EMT in the regulation of CD147. Similarly, one study focusing on the renal fibrosis in the unilateral ureteral obstruction (UUO) mice model provides the convincing outcomes of Bsg/CD147 in the promoting the development of renal fibrosis by knocking out the Bsg in mice, which was consistent with our findings (11). Compared to this study, we also noticed that EMT could serve as a key downstream process involved in the regulation of CyPA/CD147 in renal fibrosis. Interestingly, we also observed the significant infiltration of mononuclear cells and highly expressed CyPA/CD147 distributed in the IF/TA of rat model with CAD. With regard to the effect of CyPA/CD147 in inflammatory responses, we tended to consider that there was a potential link of CyPA/CD147 and immunological cells infiltration during the CAD process, which still remained further analysis. Therefore, our findings supported the CD147 as a candidate target molecule for the treatment of renal fibrosis, especially in renal allograft fibrosis.

We have identified that CyPA may have a direct pro-fibrotic effect on the tubular epithelial cells and renal fibrosis. The canonical stimulation for the renal fibrosis has been recognized as TGF- $\beta 1$, TNF-a, MCP-1 and CXCL-10 $(16,17)$. Considering the important role of CD147 in the regulation of renal fibrosis, the CyPA binding to the CD147 was also noticed to be involved in the stimulation of renal fibrosis. Moreover, extracellular CyPA has been described to induce the chemotaxis of T cells, monocytes, and macrophages, which leads to the massive recruitment and infiltration of inflammatory cells in the renal allograft tissues $(10,18)$. It is well known that certain pro-fibrotic cytokines could be produced and secreted during the inflammatory response, triggering the pathogenesis of organ fibrosis. Therefore, the indirect pro-fibrotic 
effect of CyPA should also draw great attention even though the crucial role of CyPA/CD147 interaction in renal fibrosis.

Recent study has reported the positive relation of CyPA/CD147 signaling and osteoclast-related MMP-9 expression in mice inflammatory periapical lesions progression (18). Additionally, CyPA inhibitor NIM811 significantly reduced MMP-9 secretion during the differentiation process of foam cells, which also suggested the potential association of the CyPA/CD147 and MMP-9 expression. Importantly, suppressed activities of MMP-2 and MMP-9 proteins in the Bsg-/-UUO mice was observed, providing the direct evidence for the CyPA in the regulation of MMP-9 expression (11). Thus, we believed that except for the EMT process, the MMP-9-related signaling induced by CyPA/CD147 interactions also plays a key role in the extracellular matrix deposition and degradation, and consequently renal fibrosis. In the in vitro study, we used the CD147 mAb to selectively block the interactions between CyPA and CD147, and observed the attenuation of EMT and renal fibrosis, which was consistent with other studies and our in vivo study. With great regret, we could not perform the intervention of CD147 mAb in the rat CAD model or the Bsg-/- CAD model to explore and confirm the unique effect of CD147 in the renal allograft fibrosis and CAD.

\section{Conclusion}

In conclusion, our study reported the significant expressed CyPA/CD147 in the renal allograft fibrosis tissues, and suggested the potential association of CyPA/CD147 interactions with CAD. Moreover, in vitro study showed that CyPA could remarkably promote the EMT process by binding to the CD147 and triggering the intracellular MAPK signaling pathway, which contributes to the renal fibrosis. Our data provide evidence that CyPA is critically involved in the pathophysiology of renal allograft fibrosis, and CyPA/CD147 interactions may represent a target to modulate the renal allograft fibrosis and CAD.

\section{Declarations}

\section{Ethics approval and consent to participate}

The study protocol was in compliance with the principle of the Declaration of Helsinki and Istanbul. Whole study design, including animal and cell experiments, was approved by the ethics committee of Huai'an First People's Hospital. The study was carried out in compliance with the ARRIVE guidelines

\section{Consent for publication}

Written informed consent for publication was obtained from all participants.

\section{Availability of data and materials}


The data analysed during the current study are available from the corresponding author on reasonable request.

\section{Competing interest statement:}

No competing interest exists in this manuscript.

\section{Funding}

This study was supported by the program of "Innovative young talent project" of Huai'an First People's Hospital.

\section{Authors' contributions}

X. Liu and K. Liu designed the study; X. Liu, Z. Tang, and X. Jiang performed the experimental studies; T. Wang and Z. Xu performed the statistical analysis; T. Wang and L. Zhao prepared Figure 1-5; X. Liu wrote the main manuscript text; Z. Xu and K. Liu revised the manuscript; K. Liu provided the funding. All authors reviewed the manuscript.

\section{Acknowledgment:}

We sincerely thank for the technical support and consultant from the renal transplant team of department of urology, The First Affiliated Hospital of Nanjing Medical University.

\section{References}

1. Wang Z, Han Z, Tao J, Wang J, Liu X, Zhou W, et al. Role of endothelial-to-mesenchymal transition induced by TGF-beta1 in transplant kidney interstitial fibrosis. J Cell Mol Med. 2017;21(10):2359-69.

2. Zhao C, Xu Z, Wang Z, Suo C, Tao J, Han Z, et al. Role of tumor necrosis factor-alpha in epithelial-tomesenchymal transition in transplanted kidney cells in recipients with chronic allograft dysfunction. Gene. 2018;642:483-90.

3. Zhou J, Cheng H, Wang Z, Chen H, Suo C, Zhang H, et al. Bortezomib attenuates renal interstitial fibrosis in kidney transplantation via regulating the EMT induced by TNF-alpha-Smurf1-Akt-mTORP70S6K pathway. J Cell Mol Med. 2019;23(8):5390-402.

4. Wang Z, Han Z, Tao J, Wang J, Liu X, Zhou W, et al. Transforming Growth Factor-beta1 Induces Endothelial-to-Mesenchymal Transition via Akt Signaling Pathway in Renal Transplant Recipients with Chronic Allograft Dysfunction. Annals of transplantation : quarterly of the Polish Transplantation Society. 2016;21:775-83. 
5. Su H, Yang Y. The roles of CyPA and CD147 in cardiac remodelling. Experimental and molecular pathology. 2018;104(3):222-6.

6. Yang L, Zhu Q, Gong J, Xie M, Jiao T. CyPA and Emmprin play a role in peri-implantitis. Clin Implant Dent Relat Res. 2018;20(2):102-9.

7. Xue L, Su L, Xie J, Du Y, Yu X. EMMPRIN-CypA contributes to the inflammatory processes in human periodontitis through infiltrating CD68(+) inflammatory cells. Int J Clin Exp Pathol. 2018;11(8):382834.

8. Setiawan LC, van Dort KA, Rits MA, Kootstra NA. Mutations in CypA Binding Region of HIV-1 Capsid Affect Capsid Stability and Viral Replication in Primary Macrophages. AIDS Res Hum Retroviruses. 2016;32(4):390-8.

9. Huet E, Vallee B, Szul D, Verrecchia F, Mourah S, Jester JV, et al. Extracellular matrix metalloproteinase inducer/CD147 promotes myofibroblast differentiation by inducing alpha-smooth muscle actin expression and collagen gel contraction: implications in tissue remodeling. FASEB journal : official publication of the Federation of American Societies for Experimental Biology. 2008;22(4):1144-54.

10. Seizer P, Klingel K, Sauter M, Westermann D, Ochmann C, Schonberger T, et al. Cyclophilin A affects inflammation, virus elimination and myocardial fibrosis in coxsackievirus B3-induced myocarditis. J Mol Cell Cardiol. 2012;53(1):6-14.

11. Kato N, Kosugi T, Sato W, Ishimoto T, Kojima H, Sato Y, et al. Basigin/CD147 promotes renal fibrosis after unilateral ureteral obstruction. The American journal of pathology. 2011;178(2):572-9.

12. Liu X, Liu K, Wang Z, Liu C, Han Z, Tao J, et al. Advanced glycation end products accelerate arteriosclerosis after renal transplantation through the AGE/RAGE/ILK pathway. Experimental and molecular pathology. 2015;99(2):312-9.

13. Yuan W, Ge H, He B. Pro-inflammatory activities induced by CyPA-EMMPRIN interaction in monocytes. Atherosclerosis. 2010;213(2):415-21.

14. Yang C, Qi R, Yang B. Pathogenesis of Chronic Allograft Dysfunction Progress to Renal Fibrosis. Advances in experimental medicine and biology. 2019;1165:101-16.

15. Strutz F. Pathogenesis of tubulointerstitial fibrosis in chronic allograft dysfunction. Clinical transplantation. 2009;23 Suppl 21:26-32.

16. Ferrari SM, Fallahi P, Ruffilli I, Elia G, Ragusa F, Paparo SR, et al. Immunomodulation of CXCL10 Secretion by Hepatitis C Virus: Could CXCL10 Be a Prognostic Marker of Chronic Hepatitis C? J Immunol Res. 2019;2019:5878960.

17. Ozdemir BH, Ozdemir AA, Colak T, Sezer S, Haberal M. The influence of tubular phenotypic changes on the development of diffuse interstitial fibrosis in renal allografts. Transplantation proceedings. 2011;43(2):527-9.

18. Wang YQ, Zhang J, Zhu LX, Yu JJ, Liu MW, Zhu ST, et al. Positive Correlation between Activated CypA/CD147 Signaling and MMP-9 Expression in Mice Inflammatory Periapical Lesion. Biomed Res Int. 2019;2019:8528719. 


\section{Figures}

A

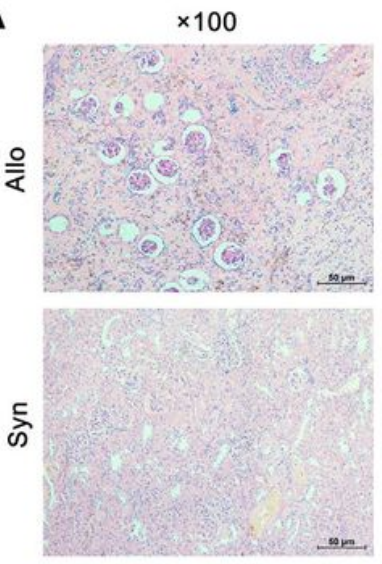

C

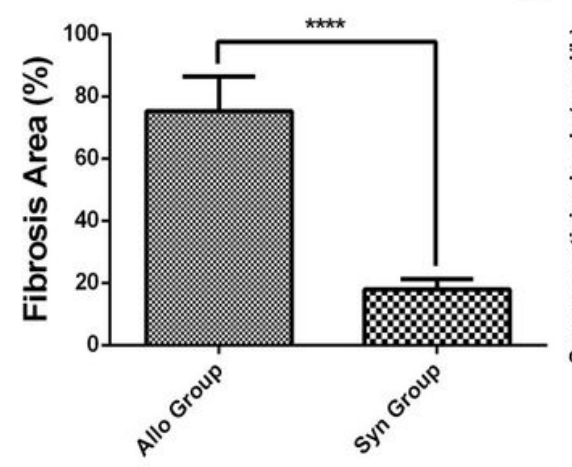

$\times 400$

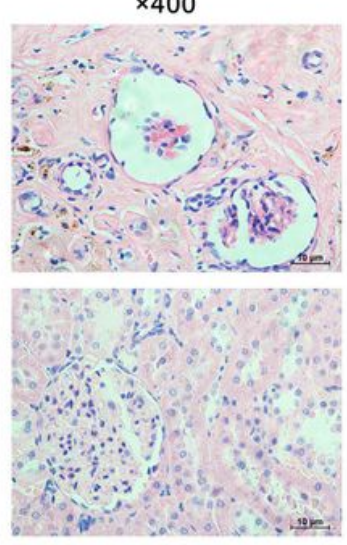

D
B

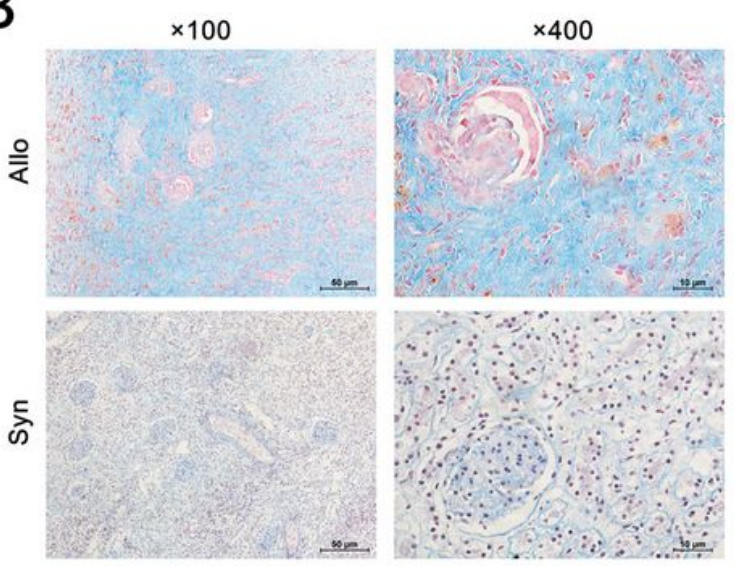

E
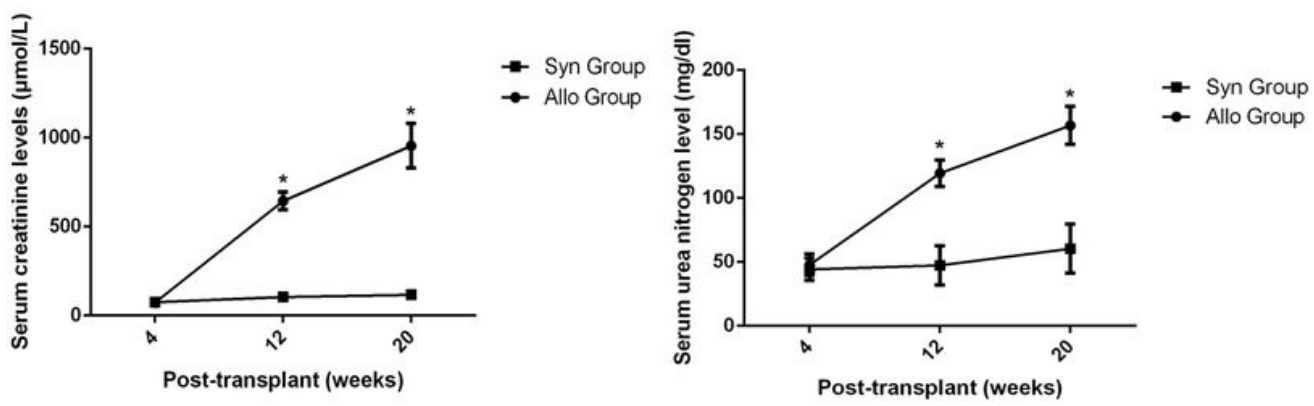

\section{Figure 1}

Establishment and identification of rat renal transplant model. We established a 20-weeks rat renal transplant model with significant chronic allograft fibrosis. The renal allograft from recipient rats were harvested and stored for further examination. HE staining (A) and Masson staining (B) were performed to identify the interstitial fibrosis and tubular atrophy in renal allograft. The quantitative analysis of fibrosis area was presented (C). Furthermore, renal allograft function was tested by serum creatinine (D) and blood urea nitrogen $(E)$. 
A

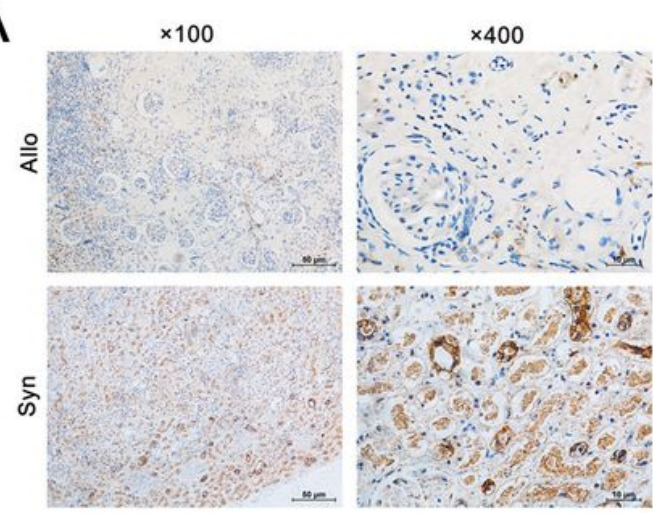

$E$

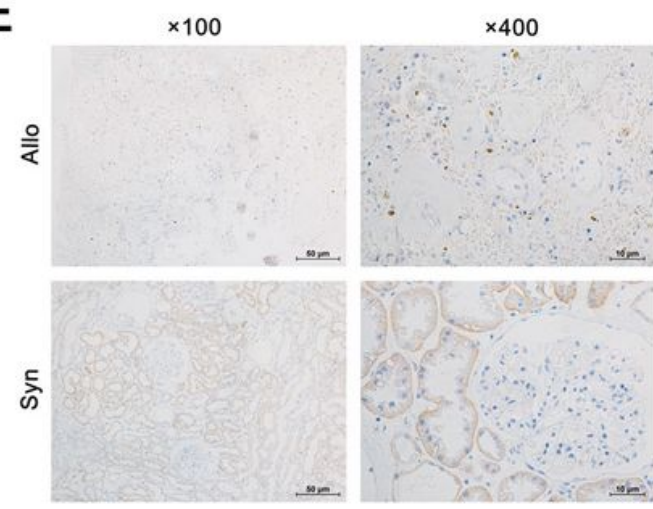

B

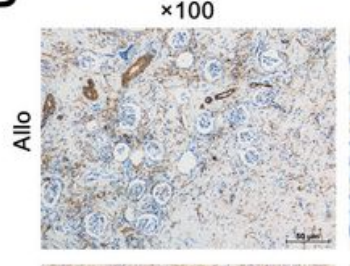

के

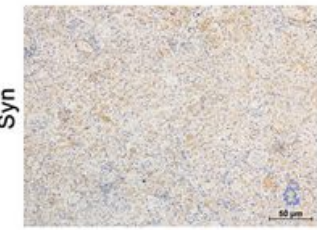

$\mathrm{F}$

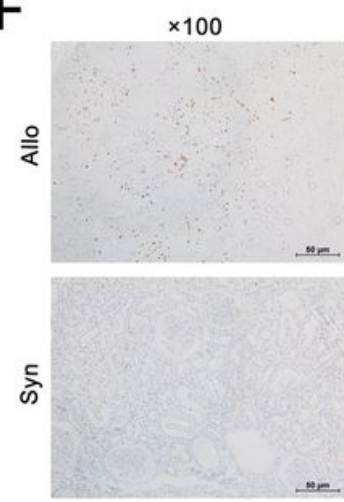

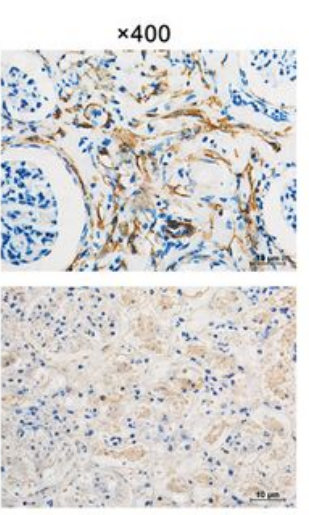

$\mathrm{C}$

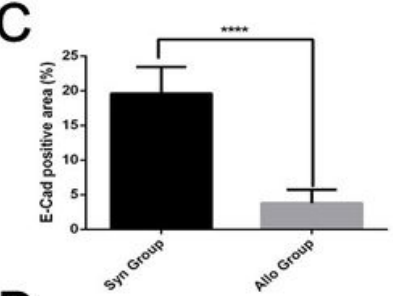

D

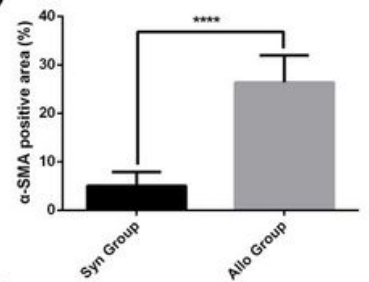

G
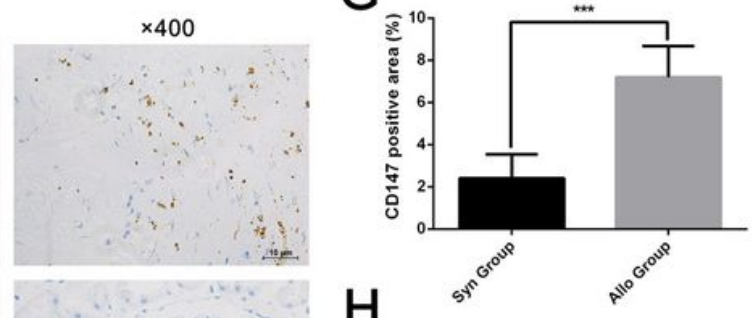

$\mathrm{H}$

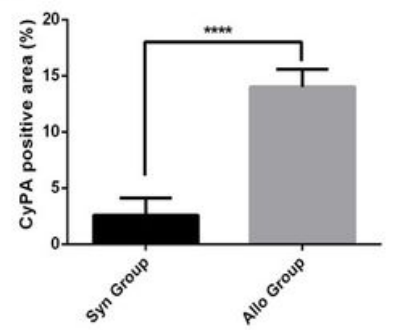

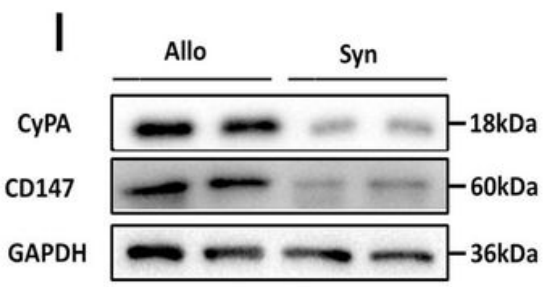
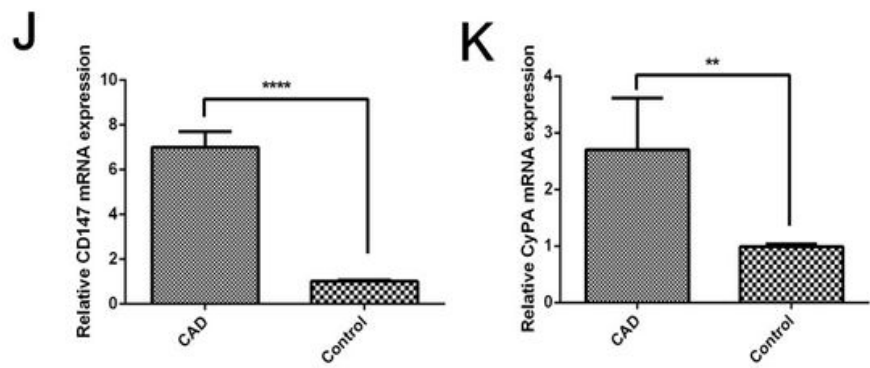

\section{Figure 2}

The expression of CyPA and CD147 in rat renal transplant model. We collected the renal allograft tissue and performed the immunohistochemistry staining of E-Cadherin (A) and a-SMA (B) to examine the occurrence of epithelial-to-mesenchymal transition in chronic allograft dysfunction, as well as the expression of CD147 (E) and CyPA (F) in renal allograft tissue. The quantitative analysis of E-Cadherin (C), a-SMA (D), CD147 (G) and CyPA (H) was presented. In addition, the protein and mRNA of CD147 and CyPA were also tested $(\mathrm{I}-\mathrm{K})$. ${ }^{\star \star \star} \mathrm{P}<0.001$ compared with the control group; $* \star \star \star \mathrm{P}<0.0001$ compared with the control group. Each experiment was repeated for at least three times. 

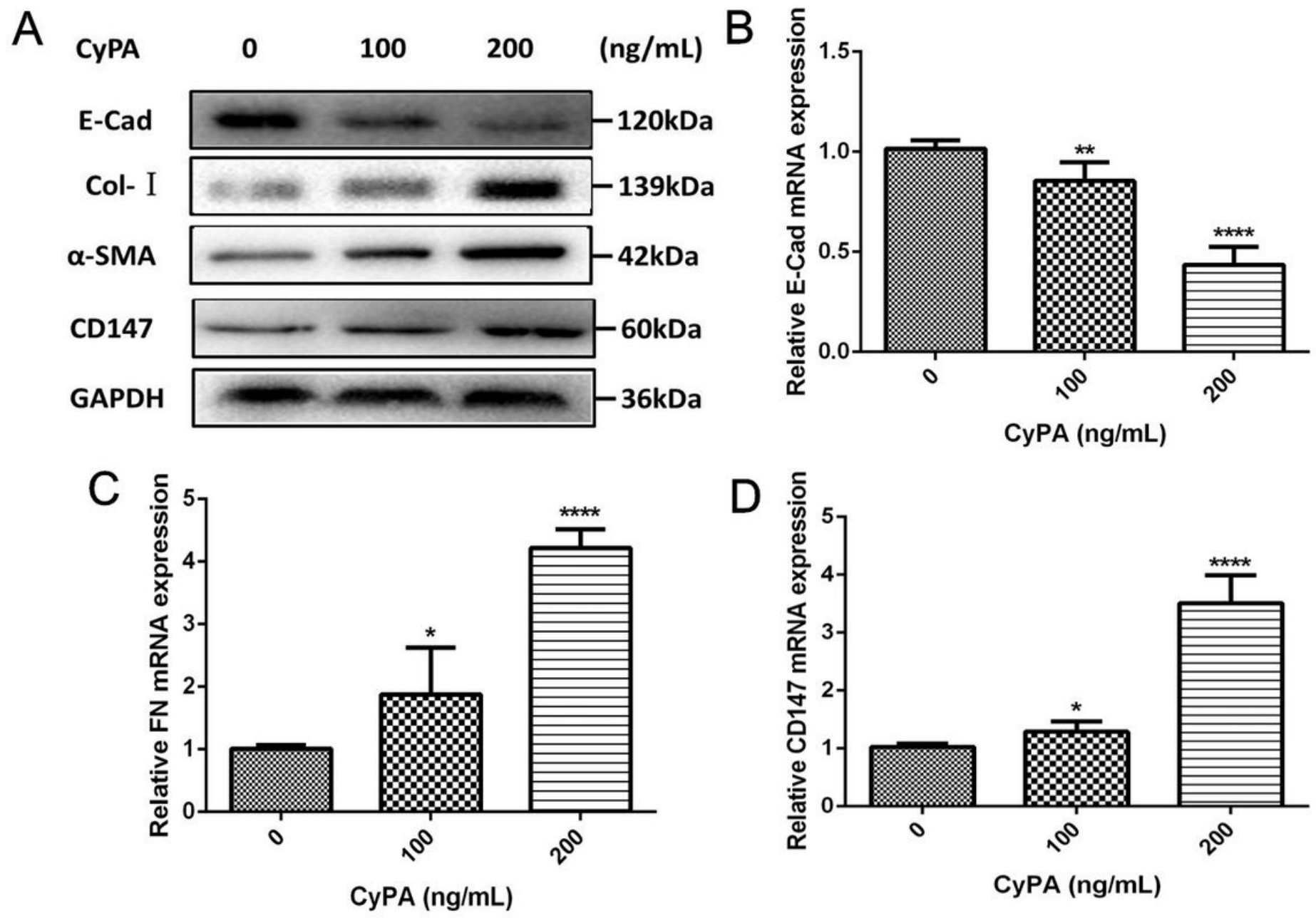

Figure 3

The effect of CyPA on CD147 and tubular fibrosis in HK-2 cells. We stimulated the HK-2 cells by various concentrations of CyPA and extracted the total protein and mRNA from cells. The CD147 protein, as well as the biomarkers related to tubular fibrosis, was tested by Western Blot assay (A). Moreover, the mRNA expression of E-Cadherin (B), Fibronectin (FN; Figure 3C) and CD147 (D) were explored by RT-PCR. *P $<0.05$ compared with the control group; ${ }^{\star \star} \mathrm{P}<0.01$ compared with the control group; $* \star \star \star \mathrm{P}<0.0001$ compared with the control group. Each experiment was repeated for at least three times. 

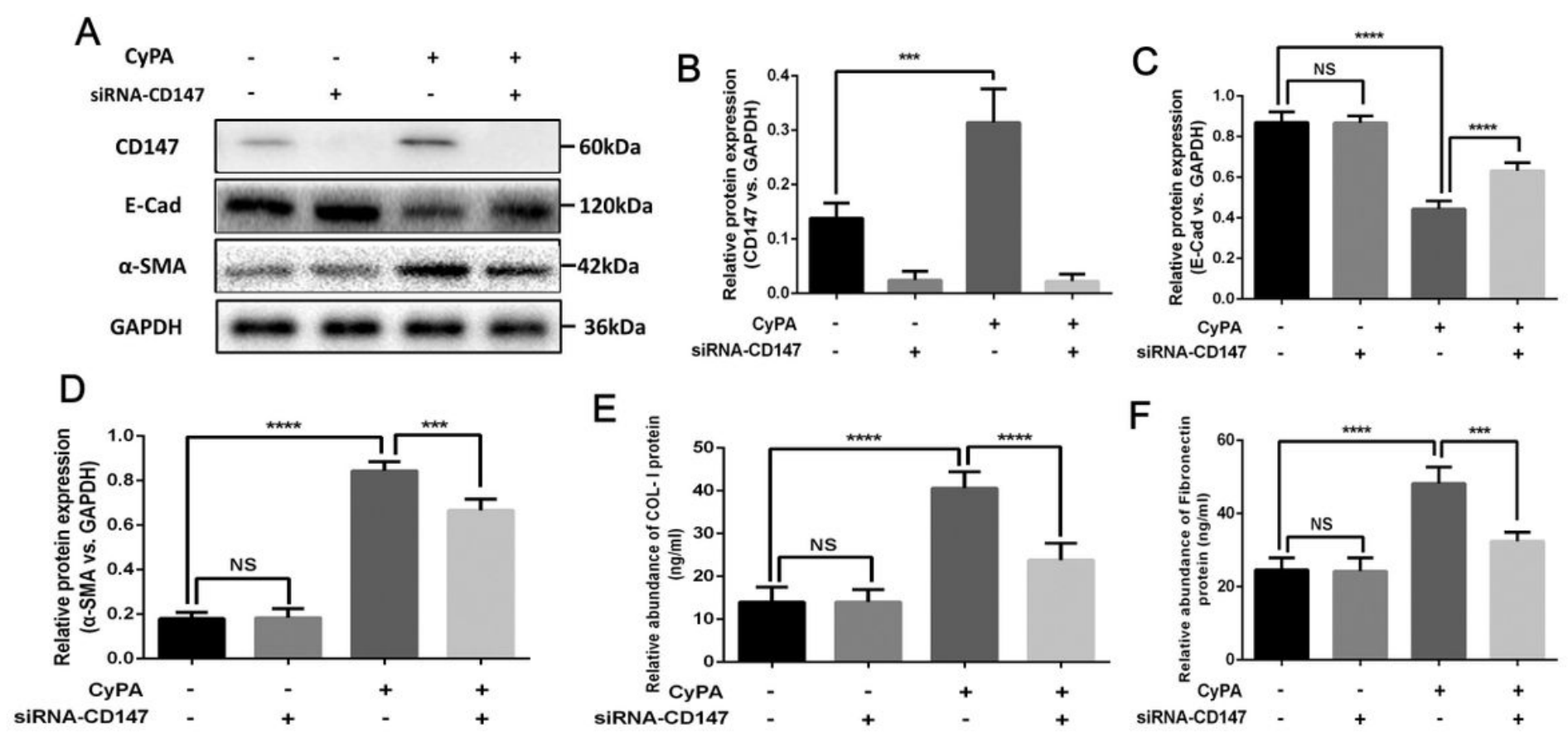

\section{Figure 4}

The identification of effect of CyPA on the CD147 expression in HK-2 cells. We conducted the siRNACD147 to slience the expression of CD147, and stimulated the HK-2 cells with or without CyPA. The quantitative analysis of CD147 (B), E-Cadherin (C), and a-SMA (D) was presented. Furthermore, the

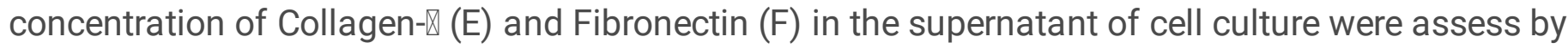
ELISA assays. NS, no significant; ${ }^{\star \star *} \mathrm{P}<0.001$ compared with the control group; ${ }^{\star \star \star \star} \mathrm{P}<0.0001$ compared with the control group. Each experiment was repeated for at least three times. 

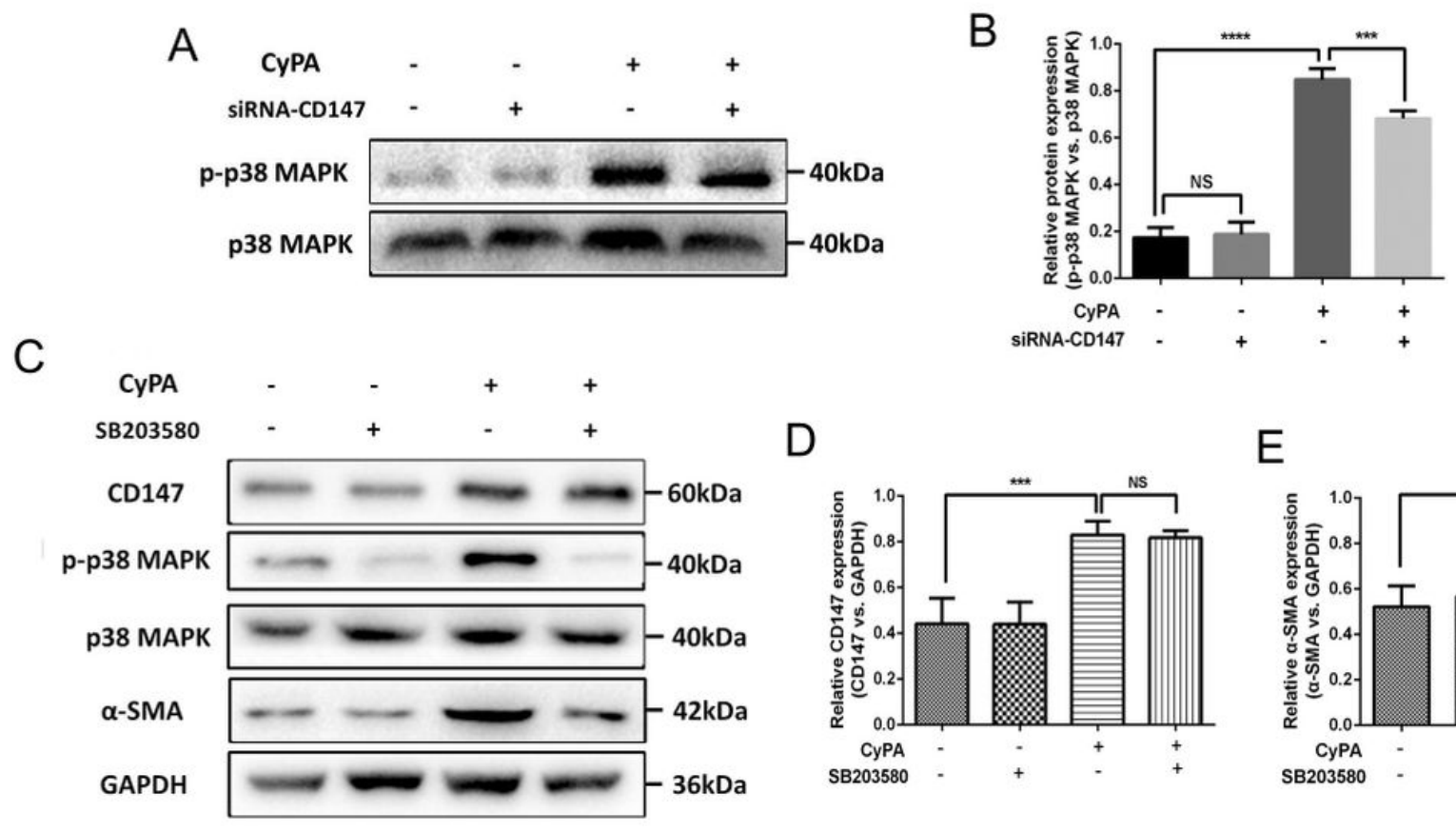

D
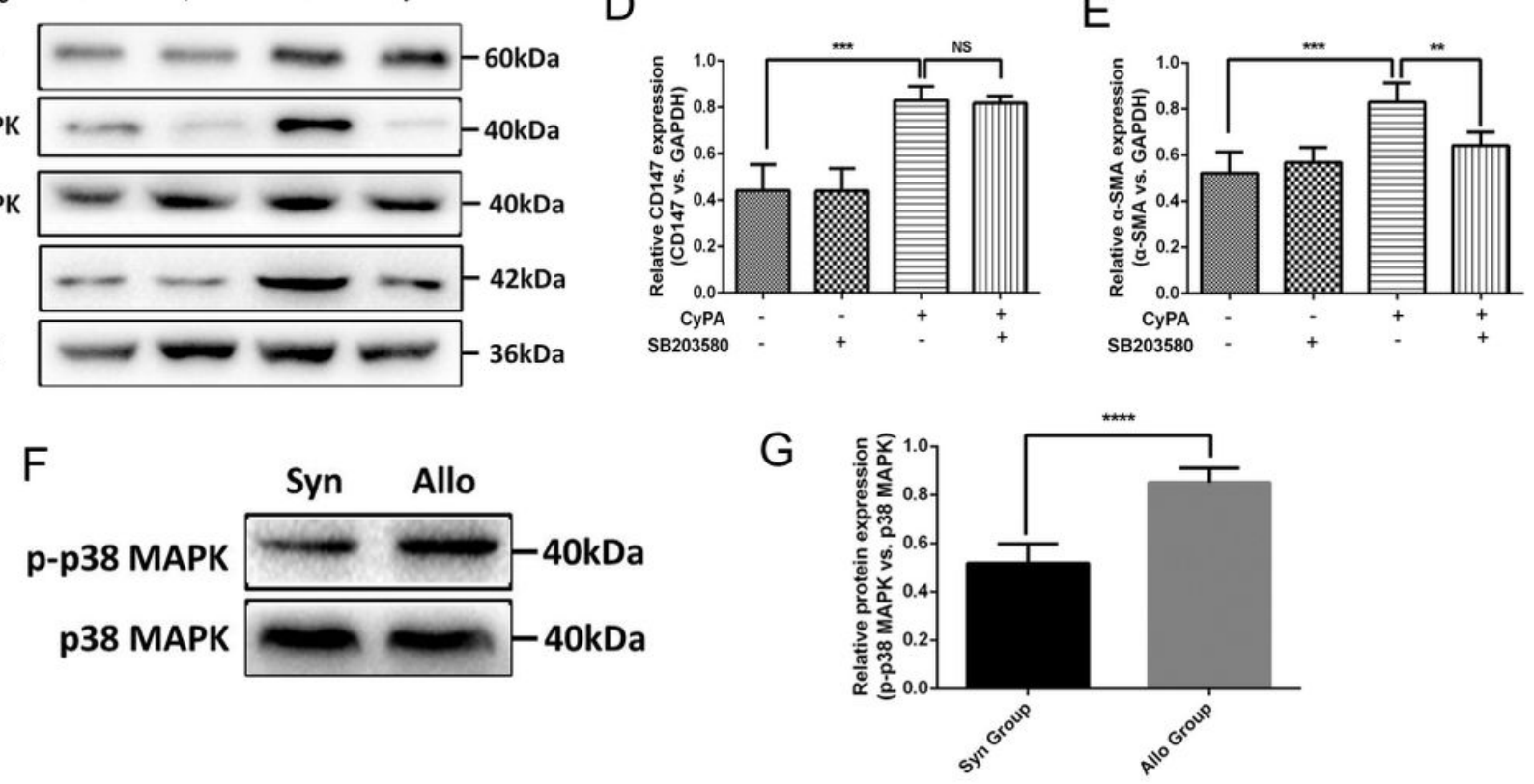

\section{Figure 5}

The study of signaling pathway involved in the CyPA/CD147 interactions in HK-2 cells. We used the siRNA-CD147 to explore the p38 MAPK signaling pathway during the CyPA/CD147 interactions in HK-2 cells (A). The quantitative analysis of phosphorylated p38 MAPK was presented in Figure 5B. Then, we selectively inhibited the expression of p38 MAPK by SB203580 and examined the expression of CD147 and a-SMA (C). The relative expression of CD147 and a-SMA were quantitatively shown (D-E). In addition, the activation of phosphorylated p38 MAPK was also tested in renal allograft tissues from rat renal transplant CAD model ( $F-G)$. NS, no significant; $* \star P<0.01$ compared with the control group; $* \star \star P<0.001$ compared with the control group; $\star \star \star \star P ~<0.0001$ compared with the control group. Each experiment was repeated for at least three times. 